\title{
Pregnancy and Skin
}

\author{
Kar Sumit • Krishnan Ajay • \\ Shivkumar Poonam Varma
}

Received: 28 March 2011 / Accepted: 8 April 2012/Published online: 28 August 2012

(C) Federation of Obstetric \& Gynecological Societies of India 2012

\begin{abstract}
Introduction Skin changes occur in about $90 \%$ pregnant women in one form or the other. The various skin changes maybe either physiological (hormonal), changes in preexisting skin diseases or development of new pregnancyspecific dermatoses. All of these dermatoses can be attributed to the profound hormonal, vascular, metabolic, and immunological changes occurring during pregnancy.

Classification Pregnancy-specific dermatoses have now been classified into dermatoses which are definitively associated and dermatoses with uncertain association with pregnancy. Though most of these skin dermatoses are benign and resolve in postpartum period, a few can risk fetal life and require antenatal surveillance. Most of the dermatoses of pregnancy can be treated conservatively but a few require intervention in the form of termination of pregnancy.

Conclusion Careful history taking and examination will help us to identify each condition clinically and appropriate
\end{abstract}

Kar S. ( $₫)$, Professor and Head

Krishnan A., Assistant Professor

Department of Dermatology, Venereology \& Leprosy, Mahatma

Gandhi Institute of Medical Sciences (MGIMS), Sevagram,

Wardha, Maharashtra 442012, India

e-mail: karmgims@gmail.com

Shivkumar P. V., Professor

Department of Obstetrics and Gynaecology, Mahatma Gandhi Institute of Medical Sciences (MGIMS), Sevagram, Wardha, Maharashtra 442012, India management can be instituted for the well-being of the mother and the fetus.

Keywords Dermatoses · Pregnancy · Skin

\section{Introduction}

Skin changes occur in more than $90 \%$ pregnant women in one form or the other [1]. During pregnancy, there is an advent of a new endocrine organ, the placenta. The fetoplacental unit synthesizes pregnenolone and progesterone. Pregnenolone is converted by the developing fetal adrenal to dehydroepiandrosterone, which is returned to the placenta to be aromatized to estriol. The parathyroid glands also hypertrophy, leading to a tendency toward low serum calcium levels. This reduction in calcium levels may have an important role in the pathogenesis of impetigo herpetiformis during pregnancy.

During pregnancy, the maternal immune response is extensively altered to allow the fetus to attach to the mother. Cytokine profile is altered, leading to a tendency toward the $\mathrm{Th}_{2}$ cytokines (IL-4, IL-5, IL-10, IL-13), which favor the maintenance of fetal survival. In postpartum period, there is elevation in the levels of $\mathrm{Th}_{1}$ cytokines (IL-2, TNF- $\alpha$, IFN- $\gamma$ ).

Hormonal changes during pregnancy also lead to an overall preference of the Th2 cytokine profile [2]. Estrogen suppresses IL-2 production while progesterone promotes the production of Th2 cytokines like IL-4, IL-5, and IL-10. 
Progesterone has an inhibitory effect on TNF- $\alpha$ secretion and glucocorticoid levels increase steadily during pregnancy. It inhibits the IL- $1, \mathrm{IL}-2$, TNF- $\alpha$, and IFN- $\gamma$ productions and stimulates IL-10, IL-4, and IL-13 syntheses.

Skin changes occurring during pregnancy can be mainly classified into four groups as shown in the Table 1. The most common changes that are seen in the Indian scenario are pigmentary changes in the form of melasma and pruritus and secondary skin changes.

Table 1 Classification of skin changes occurring during pregnancy

1. Physiological changes
Pigmentary changes
Hair and nail changes
Connective tissue changes
Vascular changes
Glandular changes

2. Specific dermatoses of pregnancy

Atopic eruption of pregnancy

Polymorphic eruption of pregnancy

Pemphigoid gestationis

Intrahepatic cholestasis of pregnancy

3. Uncertain dermatoses of pregnancy

Linear IgM disease of pregnancy

Papular dermatitis of pregnancy

Pustular psoriasis of pregnancy

Autoimmune progesterone dermatitis of pregnancy

4. Dermatological conditions modified by pregnancy

Conditions improving

Allergic contact dermatitis
Fox-Fordyce disease
Hidradenitis suppurativa
Psoriasis (2/3 improves)
Systemic sclerosis
Systemic lupus erythematosus
Conditions worsening
Atopic dermatitis
Urticaria
Hereditary angioedema
SLE (with renal involvement)
Hansen's disease
Malignant melanoma
Neurofibroma
Dermatomyositis
Eosinophilic granuloma
Pemphigus vulgaris
Mycosis fungoides
Acrodermatitis enteropathica
Porphyria cutanea tarda

\section{Physiological Skin Changes in Pregnancy}

\section{Pigmentation}

It is one of the earliest signs of pregnancy. Generalized hypermelanosis may rarely develop, and its occurrence may sometimes suggest hyperthyroidism. Freckles, nevi, and recent scars darken and even enlarge during pregnancy. It was earlier postulated that increased $\alpha$ - and $\beta$-MSH from the pituitary was the causative factor. However, it has now been proved that estrogen increased the output of melanin by the melanocytes and also that the effect of estrogen is augmented by progesterone.

Also, the placenta is rich in bioactive sphingolipids, which induce melanogenesis by upregulating various melanogenic enzymes-tyrosinase and tyrosinase-related proteins 1 and 2 .

There is usually a mild generalized pigmentation, most marked in areas that have already slightly darker than the surrounding skin like the nipples, areola, periumbilical skin, neck, upper back, and midline of the abdomen. Darkening of the skin adjoining the areola produces secondary areola, linea alba turns dark to become linea nigra especially in dark-complexioned individuals. This is often accompanied by the displacement of the umbilicus to the right, known as the "ligamentum teres sign." Other areas that become darkened are the areas of friction such as the medial thighs, the perineum, and the axillae. The affected sites usually lighten after delivery, but do not return to their actual previous color. Melasma or chloasma gravidarum occurs in about $45-75 \%$ of pregnant women [3]. Group B natural pigmentary demarcation lines occur on the limbs during pregnancy. These are borders of abrupt transition between more deeply pigmented skin and that of lighter pigmentation.

Management of the pigmentary disorders during pregnancy involves mainly reassurance and asking the patient to avoid sun exposure, as the hormonal cause of the pigmentation persists throughout the pregnancy and the mainstay therapy of the hyperpigmentation is contraindicated during pregnancy.

Hair

Hirsutism and accompanying acne can be found in many pregnant women especially toward the term. Scalp hair becomes fuller during pregnancy which is due to increase in the mean diameter of the scalp hair. Mean percentage of anagen hairs increases from the normal $85-95 \%$ by the second trimester which is due to estrogens prolonging the anagen phase and also the slow conversion of hair from the anagen to the telogen phase. After the delivery, there is accelerated conversion from anagen to telogen, and this 
results in hair fall starting from 70 to 80 days postpartum. Although complete hair regrowth usually occurs, the hair may not be as abundant as it was before. Rarely a malepattern baldness and hypotrichosis are seen especially in women with a tendency toward androgenetic alopecia.

\section{Nails}

Nail growth is generally increased during pregnancy. Nails become more brittle and soft. Distal onycholysis and subungual hyperkeratosis may occur. Beau's lines develop after delivery. Usually, the nail changes are benign, and reassurance and promotion of good nail care, avoiding any external nail sensitizers, will take care of the problems.

\section{Physiological Connective Tissue Changes During Pregnancy}

Striae distensae appear in $90 \%$ of pregnant women from the sixth to seventh month as purple streaks. A strong association between striae gravidarum and the presence of pre-existing breast and thigh striae have been found [4]. It occurs as a result of combination of distension and genetic and adrenocortical activities, which leads to intradermal tears of collagen. Preventive measures by different topical therapies are controversial [3]. Optional postpartum treatments include tretinoin, excimer laser, and surgical excision.

Molluscum fibrosum gravidarum and Acrochordons are identical to skin tags. They occur from the fourth to sixth month of pregnancy and usually disappear after parturition.

\section{Physiologic Vascular Changes During Pregnancy}

Vascular spiders or spider nevi are seen mostly in areas of skin drained by the superior vena cava, the neck, throat, face (particularly around the eyes), upper chest, arms, and hands. They appear as small, flat, or slightly raised lesions with a central, faintly pulsating, red punctum associated with small, radiating, telangiectatic vessels, and surrounding erythema.

Palmar erythema, hemangiomas, cutis marmorata, purpura, petechia, edema, carpal tunnel syndrome, varicosities, hemorrhoids, pregnancy gingivitis, and granuloma gravidarum or pregnancy epulis are the other vascular changes seen in pregnancy.

\section{Physiologic Glandular Changes During Pregnancy}

Eccrine gland activity generally increases during pregnancy, often leading to hyperhidrosis, miliaria, and dyshidrotic eczema. Apocrine gland activity usually decreases during pregnancy. Sebaceous gland function increases.

\section{Key Points}

Reassurance and counseling of the patient needs to be done. Patient cautioned against using any over-the-counter medications for pigmentation and other changes.

No need for any invasive investigations or procedures at this stage.

\section{Skin Disorders Affected by the Pregnancy}

A variety of skin disorders are affected by pregnancy mainly as a result of either the patients stopping the medications altogether by herself as soon as she knows that she is pregnant or due to the immunological changes occurring as a result of pregnancy, i.e., $\mathrm{T}_{\mathrm{H} 2}$ response is more during pregnancy and $\mathrm{T}_{\mathrm{H} 1}$ response is more in the postpartum period.

Allergic contact dermatitis, Fox-Fordyce disease, hidradenitis suppurativa, psoriasis (2/3 improves rest worsens), systemic sclerosis (may improve or no change), and systemic lupus erythematosus [5] (without renal involvement) improves clinically during pregnancy.

Atopic dermatitis [6], Urticaria (progesterone induced or premenstrual), hereditary angioedema, SLE (patients with renal involvement), Hansen's disease, malignant melanoma, neurofibroma (increased incidence of invasion of neurofibroma into the vessel wall), dermatomyositis, eosinophilic granuloma, pemphigus vulgaris [7], mycosis fungoides, acrodermatitis enteropathica and porphyria cutanea tarda-all these will worsen during the pregnancy.

\footnotetext{
Key Points

A previous history of skin disorders should always be elicited from the patients presenting with any skin manifestation during pregnancy.

Common conditions like neurofibroma and atopic dermatitis aggravates during pregnancy or may present for the first time in pregnancy.

Any therapy for a pre-existing skin condition should be modified after consulting with a dermatologist.
}

\section{The Specific Dermatosis of Pregnancy}

\section{Classification}

The first classification of dermatoses of pregnancy was proposed by Holmes and Black in 1983 and included four skin conditions: (1) Pemphigoid gestationis (PG, syn. herpes gestationis), (2) Polymorphic eruption of pregnancy (PEP) (syn., pruritic urticarial papules and plaques of pregnancy [PUPPP]), and (3) Prurigo of pregnancy (PP), 
and (4) Pruritic folliculitis (PF) of pregnancy [8]. The second, proposed by Shornick in 1998 [9], included intrahepatic cholestasis of pregnancy (ICP) in addition to $\mathrm{PG}$, PEP, and PP. The most recent rationalized classification has been proposed by Ambros-Rudolph et al. in 2006 [10] after their retrospective two-center study on 505 pregnant patients. They introduced a new entity "atopic eruption of pregnancy (AEP)" as AEP was observed to be the most common pruritic skin condition in pregnancy in their study. They included three conditions-eczema in pregnancy (EP), PP, and PF of pregnancy under AEP due to their overlapping features. Therefore, they presented four main conditions: (1) AEP, (2) PEP, (3) PG, and (4) ICP under dermatoses of pregnancy. While AEP starts significantly earlier, PEP, PG, and ICP present in late pregnancy. The uncertain dermatoses of pregnancy are linear: (1) IgM disease of pregnancy, (2) papular dermatitis of pregnancy, (3) pustular psoriasis of pregnancy, and (4) autoimmune progesterone dermatitis of pregnancy.

A few of the specific dermatoses are associated with fetal loss like herpes gestationis, pustular psoriasis of pregnancy, and cholestasis of pregnancy [11].

\section{Diseases Having Definite Association with Pregnancy}

\section{Pruritus Gravidarum and ICP}

The term pruritus gravidarum is used for all the conditions of pregnancy with severe pruritus and no skin lesions while prurigo gravidarum is used for cholestasis of pregnancy with no or minimal jaundice. The prevalence of pruritus in pregnancy was found to be $4.6 \%$ (23 cases) [12].

Pruritus is one of the most common dermatological symptoms in pregnancy [13]. Pruritus in pregnancy has always been challenging to the treating physician both in diagnosis and treatment. Pruritus occurs due to various causes, and it can occur any time during pregnancy. Causes for pruritus in pregnancy are numerous ranging from specific dermatoses of pregnancy to various dermatological conditions such as atopic dermatitis, urticarial, infection, and infestation or drug induced. In addition to these, various systemic diseases can also manifest with pruritus.

Cholestasis of pregnancy is the second most common cause of jaundice in pregnancy [14]. Pruritus is secondary to an increase in the concentration of bile acids in serum and within the skin. During pregnancy, there is a relative fall in the hepatic blood flow which leads to a decreased clearance of estrogens that result in an increase in biliary cholesterol concentration and secretion, and this also impairs the capacity of the liver to transport anions, such as bilirubin and bile salts [39]. Bile acids may solubilize lipid cellular membranes by their detergent properties, and release histamine and proteolytic enzymes such as lysosomal proteases which affect free nerve endings and produces itching sensation [15].

There are no primary skin lesions in this condition. It usually occurs in the third trimester and the itching is worse at night. Abdomen is the primary site, and later on the trunk and distal extremities get involved. Twenty percent of the patients will also have jaundice. Liver function tests may not show any abnormalities [16]. The most sensitive indicator is the serum bile acid level [9].

Although pruritus is not a life-threatening medical condition, it can be extremely troublesome for pregnant women. Because of potential effects on the fetus, the treatment of pruritus in pregnancy requires prudent consideration. However, any drug taken during the pregnancy has a potential teratogenic effect on the fetus. If a drug is consumed during the first trimester, it can result in severe structural fetal malformations and, in the later part of pregnancy, it can result in various functional defects or growth disorders and can also lead to minor malformations. Drugs taken during the pregnancy can also lead to after effects in the neonatal period and infancy.

The patient should ideally be put on bed rest and a lowfat diet. Topical calamine, emollients, mild potent steroids can be given $[9,16,17]$. Ion-exchange resins like cholestyramine, silymarine, and ursodeoxycholic acid (UDCA) [9] have also been found to be useful. $12 \mathrm{mg}$ of dexamethasone daily for 6 days has been used with suppression of fetoplacental estrogen production and resolution of pruritic symptoms [18]. UVB and phenobarbitones can also be used.

Among the antipruritic drugs prescribed during pregnancy, antihistamines are the commonest. At one point, the physician will have to use the antihistamines and weigh the benefits against the teratogenic effects of the antihistamines. Physicians must decide whether to select an older, better-studied antihistamine, thought to be relatively safe during pregnancy, or a newer agent that has less adverse effect on the quality of life, but has a potential teratogenic effect.

Many a time, the patient procures these agents as an over-the-scounter preparation and uses it. Various studies on the usage of antihistamines $\left(\mathrm{H}_{1}\right.$ blockers) during pregnancy, including a meta-analysis of more than 200,000 women, show no increase in the teratogenic risk of these drugs in the humans [19-22]. Certain animal studies with hydroxyzine, cyclizine, and promethazine have shown teratogenic effects, but no human teratogenic reports have so far been reported. Drugs such as dexchlorpheniramine and alimemazine (trimeprazine) were not found to be having any teratogenic effect even in animal studies. None of the antihistamines has so far been classified by the United States Food and Drug Administration (US-FDA), 
on the basis of controlled animal or human studies, as safe for use during the pregnancy (Table 2) [23].

If antihistamines have to be prescribed during pregnancy, then first generation agents should be preferred and among them chlorpheniramine, dexchlorpheniramine, and hydroxyzine, should be the first choice of agents. The patient should also be advised to drink plenty of water when taking antihistamines during pregnancy to overcome the anticholinergic side effects. They should also be advised to take immediate gynecological consultation if they find any change in the frequency of baby's movement or increased contractions after taking the drugs.

If a second generation agent has to be used, then loratadine or cetirizine should be preferred as they have been widely studied for possible teratogenic effects and have been found to be nonteratogenic till date. Both these agents are pregnancy category B agents.

In cholestasis of pregnancy, antihistamines offer little help, and may be used only as adjunctive therapy. The prognosis for the mother is excellent, and rapid resolution will usually occur at parturition. Incidence of prematurity, still birth, low-birthweight babies, intrauterine asphyxia, and postpartum hemorrhage are all associated with this condition and are due to placental anoxia. Strict surveillance and early induction is recommended.

\section{Herpes (Pemphigoid) Gestationis}

It has an incidence rate between 1:50,000 and 1:60,000. It is associated with HLA-DR3 and HLA-DR4, and usually occurs during 12 th to 24 th week of pregnancy in multigravida (first episode). Prodromal symptoms like malaise, nausea, headache, hot and cold sensations, constipation, fever, and chills often herald the onset of the eruption for several days or weeks. Preceding symptoms of severe generalized itching and burning sensations are prominent features of the disease. This is followed by the developments of

Table 2 FDA pregnancy category classification for antihistamines

\begin{tabular}{ll}
\hline Drug name & Pregnancy category \\
\hline Chlorpheniramine & B \\
Cyproheptadine & B \\
Dexchlorpheniramine & B \\
Hydroxyzine & C \\
Promethazine & C \\
Tripelennamine & B \\
Cetirizine & B \\
Fexofenadine & C \\
Loratidine & B \\
Levocetrizine & B \\
Desloratidine & C \\
\hline
\end{tabular}

papulo-vesicles and subepidermal bullae usually on the abdomen, often within or immediately next to the umbilicus, in half of the patients. In the other half, these begin with typical lesions, but in an atypical distribution (onset on the extremities or the palms and soles). Eruption is quite polymorphous and usually begins with areas of patchy erythema and subcutaneous edema. In more severe cases, small vesicles arise in a herpetiform or circinate pattern, frequently coalescing and forming tense bullae.

Interval between the onset of the eruption and the development of bullous lesions was found to be usually within 2-4 weeks. Duration of individual lesions is 1-3 weeks. Oral lesions are seen in $20 \%$ of the patients while face is involved in about $10 \%$ of the cases.

Direct immunofluorescence offers the most useful information for diagnosis. Herpes gestationis factor is directed against BPAG2, leading to complement activation and destruction of the basement membrane zone. Early urticarial, nonblistered areas show nonspecific findings of superficial and deep, dense, perivascular infiltrate of lymphocytes, histiocytes, and eosinophils, often characterized as a "reactive privasculitis." There is marked dermal edema, spongiosis, and necrosis of basal cells at the tip of the dermal papillae. The dermal infiltrate may vary from almost completely lymphocytic with few eosinophils to predominantly eosinophilic with nuclear fragmentation (nuclear dust). These features may sometimes be indistinguishable from erythema multiforme or bullous pemphigoid or dermatitis herpetiformis.

Therapy is directed at controlling symptoms (mainly to alleviate pruritus) and cutaneous disease (prevent new blister formation). Patients should be reassured that the disease is expected to clear after delivery. Mild cases (before the vesiculo-bullous stage) managed with potent topical corticosteroid creams, lotions and topical or systemic antihistamines. Prednisolone can be given in a dose of 15-40 $\mathrm{mg}$ per day in staggered doses. Steroid is tapered and kept at the minimal dose throughout pregnancy. A rapid increase in the prednisolone therapy will probably be required immediately postpartum. Other agents like azathioprine [16] and pyridoxine hydrochloride have also been found to be useful. Early delivery may be required in intractable cases [24]. Serious maternal are rarely seen. Graves's disease may develop later in few patients [25]. Increased fetal mortality is seen in up to $50 \%$ of the pregnancy cases that have been associated with fetal complications like miscarriage, still birth, and congenital anomalies. Cutaneous involvement of the newborn is seen in $5-10 \%$ of the patients.

Pruritic Urticarial Papules and Plaques of Pregnancy (PUPPP)

Also known as PEP or Besnier's prurigo, it is the commonest gestational dermatoses. It usually occurs in the 
third trimester in the primigravida-especially in association with large-for-dates babies or multiple pregnancies [9, 26]. Recurrence in subsequent pregnancies is rare, unless it is associated with twin or multiple pregnancies.

The main pathogenesis is the rapid, late, excessive abdominal distension resulting in collagen and elastic fiber damage in the striae, with subsequent conversion of nonantigenic molecules to antigenic ones, which may act as a trigger for the inflammatory skin changes [27].

It is characterized by erythematous, pruritic papules that coalesce into erythematous urticarial plaques typically developing suddenly and symmetrically on the abdomen. They are confined to the lower abdominal striae in twothirds of cases and typically sparing the periumbilical area (Fig. 1). It is rare to find the eruption above the breasts, hands, feet, or, like HG, involving the face. Aronson et al. classified the clinical features into three types: type I, for the most part urticarial papules and plaques; type II, nonurticarial erythema, papules, or vesicles; and type III, a combination of the two forms.

No specific laboratory abnormalities are seen. Most important differential is the rare urticarial form of HG. Erythema multiformae may be indistinguishable from PUPPP.

Prognosis is good, and no fetal or maternal complications have been reported. Reassurance and symptomatic treatment with emollients, antipruritic topical medications (menthol and doxepin), and systemic antihistamines are all that are needed $[9,26,28]$.

\section{Pruritic Folliculitis of Pregnancy}

It is a variant of PUPPP with evidence of folliculitis on histological examination. It occurs in the ratio of about 1:3,000 pregnancies [29] in the fourth to ninth month of pregnancy. It does not recur in the subsequent pregnancies.

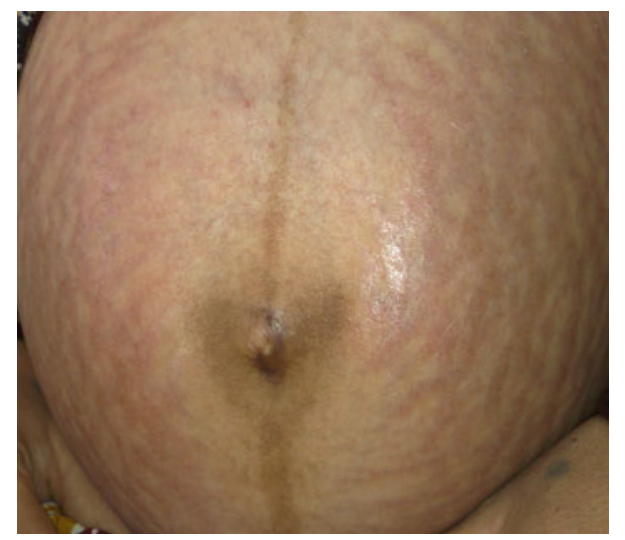

Fig. 1 PUPPP along the striae gravidarum with sparing around the umbilicus
Clinically, it presents as masses of mono-morphous 3-5$\mathrm{mm}$ follicular erythematous papules with sterile pustules, which are easily excoriated and are centered on hair follicles. It has a generalized distribution. Contrary to its name, pruritus is not a major feature.

Many cases of PF of pregnancy are misdiagnosed as acne, microbial folliculitis, or PUPPP Lesions disappear within 1 month following delivery.

Treatment is supportive with mild potent steroids and antihistamines. Topical creams containing a mixture of $10 \%$ benzoyl peroxide and $1 \%$ hydrocortisone have also been found to be useful [30]. No maternal or fetal morbidity has been reported.

\section{Prurigo of Pregnancy (PP)}

It is also known as Nurse's early onset PP. It is considered as an unusual variant of PUPPP [31]. The term "AEP" includes both PP and PF of pregnancy [10]. It occurs in the ratio of 1:300-450 pregnancies. It can occur in all trimesters, but is typically seen in the third trimester. Clinically presents as small groups of discrete, erythematous, or skin-colored papules and nodules, which are extremely itchy, resulting in excoriated lesions. It is seen primarily on the extensor surfaces of the arms and legs, on the dorsal aspects of limbs, and occasionally on the abdomen. In some cases, chest and back are involved. The nodules in PP are smaller than those in prurigo nodularis, and they are not so dark even in patients with dark-colored skin [9].

Laboratory studies reveal no abnormalities [30]. Treatment is symptomatic with moderate-to-high potent topical corticosteroids and oral antihistamines such as chlorpheniramine. Narrowband UVB 20-30 treatments have shown to be effective. No effect on the pregnancy or the newborn baby has so far been reported [32].

\footnotetext{
Key Points

Atopic eruption of pregnancy is the most common pruritic condition in pregnancy.

Cholestasis of pregnancy usually presents without any skin lesions, and the presence of primary skin lesions should raise the suspicion of other dermatoses.

Herpes gestationis usually presents with vesicles and bullae with periumbilical lesions, whereas PUPPP presents with characteristic sparing of umbilicus.

Cholestatsis of pregnancy and PUPPP occur in the third trimester, while herpes gestationis occurs in the second trimester, and atopic eczema of pregnancy can occur in any trimester.

Chlorpheniramine, dexchlorpheniramine, and hydroxyzine should be the first choice of antihistamines.

Narrowband UVB can be tried in intractable cases of pruritus.
} 


\section{Diseases with Uncertain Association with Pregnancy}

\section{Impetigo Herpetiformis}

It is also known as generalized pustular psoriasis of pregnancy. Less than 200 cases are reported in the literature. It resembles pustular psoriasis of von Zumbusch. Most patients have chronic psoriasis or have a family member with psoriasis [33], but it can also arise in a patient without previous psoriatic skin disease [16]. There is in fact no difference between IH and pustular psoriasis of von $\mathrm{Zu}-$ mbusch. Only difference is the time of appearancepregnant women developing a generalized pustular eruption were diagnosed as suffering from $\mathrm{IH}$, and nonpregnant women were diagnosed as having pustular psoriasis of von Zumbusch that usually starts late in the first trimester. Peak incidence is seen during the last 3 months. Recurrences are expected with subsequent pregnancies [33].

Primary lesion in IH is a sterile pustule. Rash usually starts as irregular erythematous macules and patches and in the margins of these arise, groups or rings of multiple, distinct pinhead sterile pustules. It is seen in the umbilicus, medial thighs, axillae, nape and anterior aspect of neck, inframammary folds and gluteal crease. The central lesions quickly broken down and exude, leaving denuded areas or becoming crusted, leaving an impetiginized appearance, from which the disease originally took its name. The eruption becomes confluent later. Mucous membranes are involved with papules and pustules, leaving painful erosions in a circinate manner. Subungual pustules may lift the nail plate. Severe systemic symptoms like fever, nausea, vomiting, and diarrhea are present. Pruritus is not a constant symptom. The disease usually resolves rapidly after parturition.

Laboratory investigations shows leukocytosis with a neutrophil increase, increased ESR, hypoalbuminemia, increased levels of blood urea nitrogen (BUN), increased levels of uric acid, hypocalcemia, and hyperphosphatemia as a manifestation of hypoparathyroidism. Histopathologically, it is impossible to differentiate this condition from pustular psoriasis.

The only other dermatitis of pregnancy having a pustule as a primary lesion is autoimmune progesterone dermatitis [34]. Clinical differentiation among IH, dermatitis herpetiformis, and pustular psoriasis may on occasion be very difficult or impossible.

Treatment is with $15-30 \mathrm{mg}$ prednisone per day of, but sometimes dosages up to $60 \mathrm{mg} /$ day are needed to prevent new pustules. Antibiotic coverage should be given. In severe cases, termination of pregnancy is advised. Prognosis for the mother is nowadays very good. But placental insufficiency may lead to stillbirths [9].
Autoimmune Progesterone Dermatitis of Pregnancy

Only a single case has been reported so far [34]. It is characterized by nonpruritic, acneiform eruption triggered by pregnancy, due to hypersensitivity to endogenous progesterone. It recurs in subsequent pregnancy [34]. It shows positive intradermal skin test to progesterone [16]. Fetal mortality was $100 \%$ in both times [34].

\section{Linear IgM Dermatosis of Pregnancy (LMDP)}

Only a single case has been reported [35]. The existence of LMDP as an entity is debated [66]. Some authors categorize it as a variant of PUPPP or PP [24]. It is characterized by intensely pruritic, red, follicular papules and pustules on the abdomen, forearms, thighs, and legs appearing after 36 weeks of pregnancy. Direct immunofluorescence testing reveals linear IgM deposits at the BMZ. No pregnancy effects were observed in the patient.

\section{Key Points}

Generalized pustular psoriasis of pregnancy generally occurs in the third trimester.

Severe systemic symptoms are present in this condition, which sometimes warrant termination of pregnancy.

Laboratory investigations like a hemogram, BUN, serum calcium levels, etc., should be done.

\section{Summary}

- Diagnosis of most of the skin disorders during pregnancy can be made by careful history taking and local examination of the skin.

- The most common pregnancy-associated skin changes observed in India are pruritus and pigmentary changes.

- Therapy for a pre-existing dermatosis, even topical medications, should be stopped after consulting with a skin specialist.

- Investigations are generally not required for majority of the skin conditions and invasive procedures like biopsy should be avoided during pregnancy.

- The antihistamines of choice during pregnancy are chlorpheniramine, dexchlorpheniramine, and hydroxyzine.

- Liberal application of topical bland emollients like petroleum jelly should be advised in all pregnancyassociated pruritus.

- Investigations like hemogram, renal and liver function tests along with serum calcium levels should be done in cases with generalized pustular psoriasis of pregnancy. 


\section{References}

1. Kumari R, Jaisankar TJ, Thappa DM. A clinical study of skin changes in pregnancy. Indian $\mathrm{J}$ Dermatol Venereol Leprol. 2007;73(2): 141 .

2. Wilder RL. Hormones, pregnancy, and autoimmune disease. Ann N Y Acad Sci. 1998;840:45-50.

3. Barankin B, Silver SG, Carruthers A. The skin in pregnancy. J Cutan Med Surg. 2002;6:236-40.

4. Chang AL, Agredano YZ, Kimball AB. Risk factors associated with striae gravidarum. J Am Acad Dermatol. 2004;51:881-5.

5. Gordon C. Pregnancy and autoimmune diseases. Best Pract Res Clin Rheumatol. 2004;18:359-79.

6. Torgerson RR, Marnach ML, Bruce AJ. Oral and vulvar changes in pregnancy. Clin Dermatol. 2006;24:122-32.

7. Goldberg NS, DeFeo C, Kirshenbaum N. Pemphigus vulgaris and pregnancy: risk factors and recommendations. J Am Acad Dermatol. 1993;28:877-9.

8. Holmes RC, Black MM. The specific dermatoses of pregnancy. J Am Acad Dermatol. 1983;8:405-12.

9. Shornick JK. Dermatoses of pregnancy. Semin Cutan Med Surg. 1998;17:172-81.

10. Ambros-Rudolph CM, Mullegger RR, Vaughan-Jones SA, et al. The specific dermatoses of pregnancy revisited and reclassified: Results of a retrospective two-center study on 505 pregnant patients. Am Acad Dermatol. 2006;54:395-404.

11. Sachdeva S. The dermatoses of pregnancy. Indian J Dermatol. 2008;53(3):103-5.

12. Shanmugam S, Thappa DM, Habeebullah S. Pruritus gravidarum: a clinical and laboratory study. J Dermatol. 1998;25(9):582-6.

13. Black MM, McKay M, Braude PR. Color atlas and text of obstetric and gynecologic dermatology. 2nd ed. London: Times Mirror International Publishers Limited; 2001.

14. Sherard GB 3rd, Atkinson SM Jr. Focus on primary care: pruritic dermatological conditions in pregnancy. Obstet Gynecol Surv. 2001;56:427-32.

15. Varadi DP. Pruritus induced by crude bile and purified bile acids. Experimental production of pruritus in human skin. Arch Dermatol. 1974;109:678-81.

16. Winton GB, Lewis CW. Dermatoses of pregnancy. J Am Acad Dermatol. 1982;6:977-98.

17. Fagan EA. Intrahepatic cholestasis of pregnancy. BMJ. 1994; 309:1243-4.

18. Hirvioja ML, Tuimala R, Vuori J. The treatment of intrahepatic cholestasis of pregnancy by dexamethasone. $\mathrm{Br} \mathrm{J}$ Obstet Gynaecol. 1992;99:109-11.
19. Stephansson O, Granath F, Svensson T, et al. Drug use during pregnancy in Sweden-assessed by the Prescribed Drug Register and the Medical Birth Register. Clin Epidemiol. 2011;3:43-50.

20. Seto A, Einarson T, Koren G. Pregnancy outcome following first trimester exposure to antihistamines: meta-analysis. Am J Perinatol. 1997;14:119-24.

21. Weber-Schoendorfer C, Schaefer C. The safety of cetirizine during pregnancy. A prospective observational cohort study. Reprod Toxicol. 2008;26:19-23.

22. Gilboa SM, Strickland MJ, Olshan AF, et al. Use of antihistamine medications during early pregnancy and isolated major malformations. Birth Defects Res A. 2009;85:137-50.

23. Lis-Swiety AD, Brzezinska-Wcislo LA. The safety of the antihistamines in dermatoses of pregnancy. Wiad Lek. 2006;59: 89-91.

24. Kroumpouzos G, Cohen LM. Dermatoses of pregnancy. J Am Acad Dermatol. 2001;45(1-19):19-22.

25. Shornick JK, Black MM. Secondary autoimmune diseases in herpes gestationis (pemphigoid gestationis). J Am Acad Dermatol. 1992;26:563-6.

26. Al-Fares SI, Jones SV, Black MM. The specific dermatoses of pregnancy: a re-appraisal. J Eur Acad Dermatol Venereol. 2001; 15:197-206.

27. Aronson IK, Bond S, Fiedler VC. Pruritic urticarial papules and plaques of pregnancy: clinical and immunopathologic observations in 57 patients. J Am Acad Dermatol. 1998;39:933-9.

28. Murray JC. Pregnancy and the skin. Dermatol Clin. 1990;8: 327-34.

29. Roger D, Vaillant L, Fignon A. Specific pruritic diseases of pregnancy. A prospective study of 3192 pregnant women. Arch Dermatol. 1994;130:734-9.

30. Black MM. Prurigo of pregnancy, papular dermatitis of pregnancy, and pruritic folliculitis of pregnancy. Semin Dermatol. 1989;8:23-5.

31. Dahdah MJ, Kibbi AG. Less well-defined dermatoses of pregnancy. Clin Dermatol. 2006;24:118-21.

32. Vaughan Jones SA, Hern S, Nelson-Piercy C. A prospective study of 200 women with dermatoses of pregnancy correlating clinical findings with hormonal and immunopathological profiles. Br J Dermatol. 1999;141:71-81.

33. Dacus JV. Pruritus in pregnancy. Clin Obstet Gynecol. 1990;33: 738-45.

34. Bierman SM. Autoimmune progesterone dermatitis of pregnancy. Arch Dermatol. 1973;107:896-901.

35. Alcalay J, Ingber A, Hazaz B. Linear IgM dermatosis of pregnancy. J Am Acad Dermatol. 1988;18:412-5. 